\title{
Linear Viscoelasticity of Weakly Hydrogen-Bonded Polymers near and below the Sol-Gel Transition
}

\author{
Milad Golkaram, ${ }^{\dagger}$ Csaba Fodor, ${ }^{\dagger}$ Evelyne van Ruymbeke, ${ }^{\ddagger 0}$ and Katja Loos ${ }^{* \dagger}{ }^{\dagger}$ \\ ${ }^{\dagger}$ Macromolecular Chemistry and New Polymeric Materials, Zernike Institute for Advanced Materials, University of Groningen, \\ Nijenborgh 4, 9747 AG Groningen, The Netherlands \\ ${ }^{\ddagger}$ Bio-and Soft Matter, Institute of Condensed Matter and Nanosciences, Université Catholique de Louvain, Croix du Sud 1, B-1348 \\ Louvain-la-Neuve, Belgium
}

\section{Supporting Information}

ABSTRACT: Supramolecular polymers bearing weak hydrogen bonds (sticker) can express outstanding dynamic properties due to their labile association. Studying the linear viscoelasticity (LVE) of this type of polymer can provide us with sufficient knowledge to design polymeric materials for applications that need dynamic properties such as self-healing. Using different compositions of flexible weak stickers, LVE analysis showed scalings corresponding to a transition from a linear precursor to a cluster. By introducing one sticker per repeating unit of the precursor polymer, the effect of sticker distribution along the
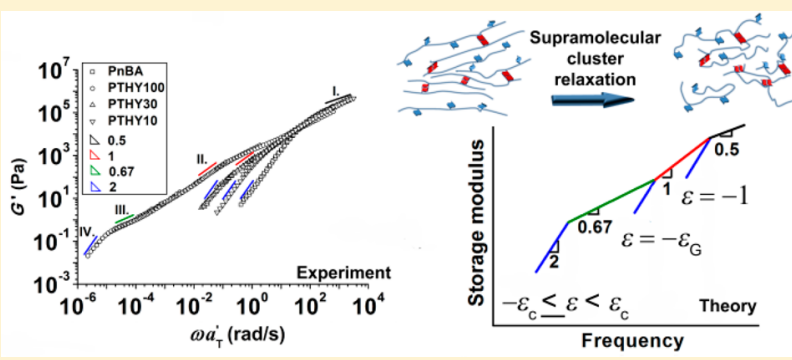
chain as well as phase separation is excluded. However, even a fully functionalized polymer could not show any network formation, whereas surprisingly, a stable cluster was formed. This proves that weakly associated networks do not dissociate rapidly and can relax as a cluster at extended time before the dissociation of stickers can lead to the relaxation of linear analogous (slow kinetics similar to strong physical or even chemical bonds.) On the other hand, the absence of a gel even in fully sticker-functionalized polymers shows that the weakness of these polymers can be described as their weakness in complete association (thermodynamically not favored).

\section{INTRODUCTION}

Introduction of supramolecular moieties to polymer chains that can readily stick to each other (called stickers) results in unique material properties, such as self-healing and shape memory. ${ }^{1,2}$ These properties mainly originate from the dynamic behavior of the introduced moieties and can be tuned by using different interactions, such as strong ionic, ${ }^{3}$ metal complex, ${ }^{4}$ or hydrogen bonding, ${ }^{5-8}$ which increase both the rheological relaxation time and the plateau modulus of the sample. The linear viscoelasticity (LVE) analysis of polymers based on acrylic acid (AA), carboxyethyl acrylate (CEA), and acrylamidopyridine (AP) was carried out by Anthamatten and co-workers.' However, the association in the corresponding polymers was unsuccessful, evident from their Rouse-like behavior. Monomers bearing weak hydrogen bonding functionalities such as thymine and adenine have been studied extensively, and it was observed that the flexible spacers introduced to the nucleobases lead to an improved capacity of self-assembly, which was attributed to the ability of each sticker to gain a suitable conformation for coupling with the nearest moiety. $^{10}$

The modeling of viscoelasticity near the sol-gel transition for the supramolecular polymers with side stickers is limited to the seminal works of Rubinstein and Semenov, ${ }^{11,12}$ whereby the dynamics of supramolecular polymers were studied, and a new theory for the gelation of associating polymers was introduced. The initial theory developed in 1998 was a meanfield (MF) approach to examine the viscoelasticity of associating polymers, which was later used to predict the sol-gel transition in (unentangled) randomly sulfonated polystyrene (SP). ${ }^{13}$ Although this model could explain the viscoelasticity of the polymers both above and below the gel point, it failed to predict the behavior close to the gel point. Therefore, the theory was further modified ${ }^{3,8,14}$ by considering a transition from MF to critical percolation (CP) known as the Ginzburg point, when the large sol clusters newly formed (through association of stickers) are not overlapping in space. ${ }^{3}$ This modified model was applied to the polymers containing strong stickers, for which it was developed. However, scalings were only partially been observed due to (1) phase separation, (2) distribution of stickers along the chain, and (3) high affinity of the used stickers, which makes it hard to access the Ginzburg point where the transitions of the scalings are extremely sensitive to the degree of association.

The LVE of supramolecular polymers based on poly $(n$-butyl acrylate) (PnBA) has been investigated previously. ${ }^{15,16}$ Despite their hypothesis that the aggregation visible in the LVE of PnBA is due to partial hydrolysis of butyl acrylate groups, it has

Received: April 11, 2018

Revised: June 13, 2018

Published: June 27, 2018 
been shown by Beiner et al. that nanophase separation can occur in intact PnBA due to incompatible main and butyl side chains. ${ }^{17}$

Moreover, it has been shown that the presence of a terminal slope shallower than 1 and 2 for $G^{\prime \prime}$ and $G^{\prime}$ is due to (1) a random distribution of the stickers along the chain, (2) a disproportionate amount of stickers in different chains, and (3) polydispersity in the chain length, leading to a distribution of terminal relaxation times. ${ }^{18,19}$ The authors therefore could not observe a terminal slope of 1 and 2 for $G^{\prime \prime}$ and $G^{\prime}$, corresponding to the Maxwell relaxation, and strived to exclude this effects via including the sticker polydispersity in the modeling.

In this study using $\mathrm{nBA}$ and thymine-functionalized $\mathrm{nBA}$ (THY) we first investigate the viscoelasticity near the sol-gel transition with varying the sticker composition. Followed by using a fully functionalized polymer, the effect of butyl side chain aggregation as well as sticker distribution is excluded to study a pure contribution from the weak stickers in the corresponding cluster/gel.

\section{BACKGROUND}

For the detailed description of the theory we encourage reading the paper by Chen et $\mathrm{al}^{3}$ For a chemical sol/gel, the gelation point can be predicted from the growth of the sol, generation by generation to an infinite size, and can be written as eq 1 :

$$
p_{c}=1 /(N-1)
$$

where $N$ is the functionality, $N-1$ corresponds to the available (potential) functionalities for the next generation as one is already taken by the previous generation, and $p_{c}$ represents the average number fraction of stickers assuming all the stickers are in the bonded state, so that having only slightly more than 1 sticker per chain in average for sufficiently long chains $(N \gg 1)$ leads to gelation. In the case of stickers with weak hydrogen bonding, the full association of the stickers might not be the case as is discussed later in the Results and Discussion. The extent of gelation is written as

$$
\varepsilon=\left(p-p_{c}\right) / p_{c}
$$

where $\varepsilon=0$ and 1 correspond to the gel point (gel content $\sim 0 \%$ ) and full gelation (gel content $\sim 100 \%$ ), respectively, $p$ being the degree of reaction. The summary of the theory is depicted in Figure $3 \mathrm{~b}$. For the first regime with $\varepsilon=-1$ the behavior of an unentangled linear polymer (the precursor chain) is described with the $G^{\prime} \sim \omega^{n}$ (where $n=0.5$ and 1 before and after the Rouse relaxation time).

$$
\begin{aligned}
& \text { precursor chain molecular weight: } M_{X}=M_{0} N_{X} \\
& \text { precursor chain size: } R_{X}=N_{X}^{1 / 2} b
\end{aligned}
$$

precursor chain Rouse relaxation time: $\tau_{X}=\tau_{0} N_{X}{ }^{2}$

with $b$ the segmental size, $N_{X}$ the number of segments, $M_{0}$ the segment molecular weight, and $\tau_{0}$ the relaxation time of one segment.

For the second regime $\left(-1<\varepsilon<-\varepsilon_{\mathrm{G}}\right.$, with $\varepsilon_{\mathrm{G}}$ being the extent of the reaction at Ginzburg point), clusters are formed via MF percolation, and the relaxation time is governed by the Rouse relaxation of the cluster leading to $G^{\prime} \sim \omega^{n}$ (where $n=$ 1) before the terminal relaxation.
The MF theory holds until the Ginzburg point, where the characteristic clusters no longer overlap and the MF scaling no longer holds. Therefore, the third regime, corresponds to $-\varepsilon_{\mathrm{G}}$ $\leq \varepsilon<-\varepsilon_{\mathrm{c}}$ where MF and critical percolation (CP) apply in shorter and longer times than $\tau_{X} N_{X}$, wherein $G^{\prime} \sim \omega^{n}$ (where $n$ $=1$ and 0.67 for MF and CP). For longer time scales a Maxwell relaxation $(n=2)$ can be seen which belongs to the relaxation of the entire cluster.

In the fourth regime $-\varepsilon_{\mathrm{c}} \leq \varepsilon<\varepsilon_{\mathrm{c}}$, a time called "effective breakup time" appears, which was first introduced by Rubinstein and Semenov, ${ }^{11,12}$ indicating the time in which the cluster breakup into two comparable clusters via breaking of the stickers in the time scales longer than their lifetime. Therefore, there is a competition between the time scale for the dissociation of the cluster into two clusters $\left(\tau_{\text {life }}=\tau_{s} \varepsilon\right)$ and the relaxation of the characteristic mother cluster $\left(\tau_{\text {char }}=\right.$ $\tau_{X}|\varepsilon|^{-3}$ ), with $\tau_{X}$ and $\tau_{\text {life }}$ being the Rouse relaxation time of the precursor chains and the effective breakup time, respectively. By approaching the gel point, the strands reach an infinite molecular weight with longer relaxation times, but having shorter lifetime, as there are more stickers available to break. One can consequently calculate $\tau_{\mathrm{c}}$ as

$$
\tau_{\mathrm{c}}=\tau_{X}{ }^{1 / 4} \tau_{\mathrm{s}}{ }^{3 / 4} \quad\left(\text { if }-\varepsilon_{\mathrm{c}} \leq \varepsilon<\varepsilon_{\mathrm{c}}\right)
$$

where $\tau_{s}$ is the lifetime of the stickers. For $1<\varepsilon$ the gel strands are very dense, and the breakup of one strand does not lead to a full relaxation of the precursor chains, which leads to a plateau in the storage modulus as has been described by the sticky Rouse model. ${ }^{12}$

\section{EXPERIMENTAL SECTION}

3.1. Materials. $n$-Butyl acrylate (nBA) was purchased from Aldrich and passed through neutral alumina column before use. $\alpha, \alpha^{\prime}$ Azobis(isobutyronitrile) (AIBN, Fluka, 99\%) was recrystallized from methanol. Thymine, S,S-dibenzyl trithiocarbonate (DBTTC), 1,4butanediol diacrylate, triethylamine (TEA), acetone- $d_{6}$, dimethyl sulfoxide- $d_{6}$, and 2,6-di-tert-butyl-4-methylphenol (BHT) were purchased from Aldrich and used without further purification. Hexane, chloroform, tetrahydrofuran (THF), methanol and $\mathrm{N}, \mathrm{N}$ dimethylformamide (DMF, anhydrous) were purchased from Fisher Scientific and used as received. Thymine-functionalized nBA (THY) was synthesized according to the literature. ${ }^{10}$

3.2. Synthesis of PTHYi. Synthesis of PTHY $i$ was carried out via polymerization of the required amount of THY and $\mathrm{nBA}$ initiated by AIBN and DBTTC as the chain transfer agent. To a Schlenk tube containing a magnetic stirrer, DBTTC, and AIBN in DMF, the required amounts (see Table S1) of $\mathrm{nBA}$ and THY were added followed by four freeze-pump-thaw cycles. Then, the reaction mixture was inserted in a preheated oil bath of $70^{\circ} \mathrm{C}$ and stirred for 7 h. Subsequently the reaction mixture was precipitated in a methanolwater mixture and recovered via centrifugation. The polymers were dried under vacuum and yielded the desired product (Table S1).

3.3. Characterization. ${ }^{1} \mathrm{H}$ NMR spectra were recorded at room temperature on a Varian VXR $400 \mathrm{MHz}\left({ }^{1} \mathrm{H}: 400 \mathrm{MHz}\right)$ spectrometer using deuterated solvents. Chemical shifts $(\delta)$ are reported in ppm, whereas the chemical shifts are calibrated to the solvent residual peaks. Gel permeation chromatography (GPC) measurements were performed in THF at $25{ }^{\circ} \mathrm{C}(1 \mathrm{~mL} / \mathrm{min})$ on a Spectra-Physics AS 1000, equipped with PLGel $5 \mu \mathrm{m} \times 30 \mathrm{~cm}$ mixed$\mathrm{C}$ columns. Universal calibration was applied using a Viscotek $\mathrm{H} 502$ viscometer and a Shodex RI-71 refractive index detector. The GPC was calibrated using narrow disperse polystyrene standards (Polymer Laboratories). Melt rheology was carried out via a TA Instruments AR 1000 under nitrogen flow, and $25 \mathrm{~mm}$ parallel plate geometries and interplate gap of $0.8-1 \mathrm{~mm}$ were used in all cases. Samples were vacuum-dried overnight before use. All measurements were performed 
Scheme 1. Synthesis of PTHYi (Co)polymers via RAFT Copolymerization of nBA and THY
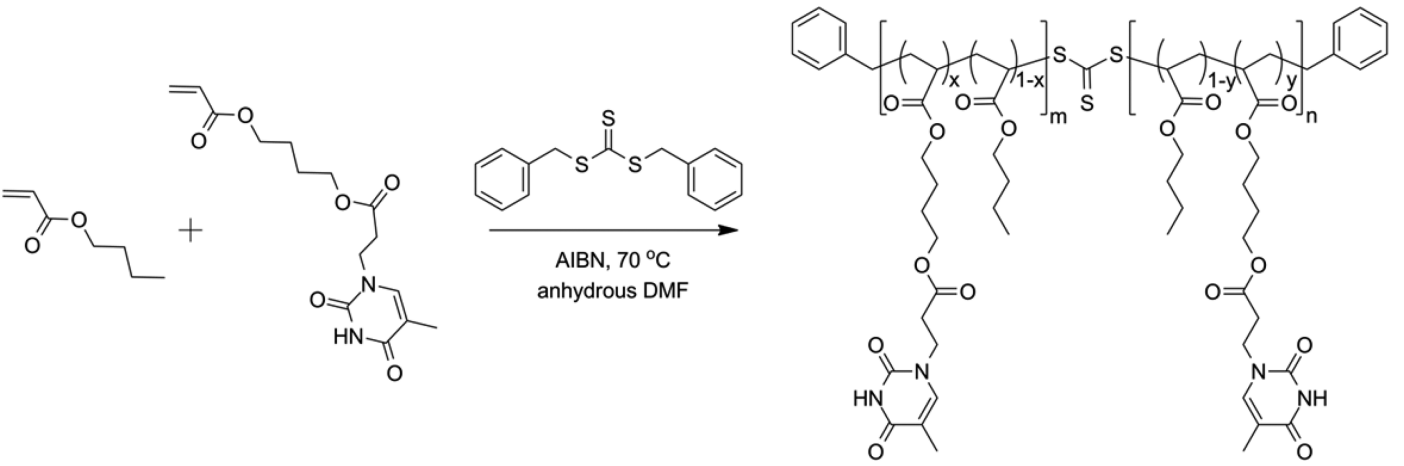

in the linear viscoelastic regime, determined via torque sweep measurements. Frequency sweeps were carried out at different temperatures (for PTHY100 they were 40, 45, 55, 65, 75, 85, and $90{ }^{\circ} \mathrm{C}$ ), and for the reproducibility of the data the measurement at $T_{r}$ was repeated. In all cases, the repeated frequency sweeps were the same within $5 \%$ error.

\section{RESULTS AND DISCUSSION}

RAFT copolymerization of $\mathrm{nBA}$ and THY led to a variety of poly(acrylic thymine-co- $n$-butyl acrylate)s (PTHY $i, i=(x+y)$ $\times 100$, representing the molar percentage of thymine moieties in the polymer) with different contents of thymine. Polymers with dispersities $(\boxplus)$ of about $1.2-1.3$ were obtained for the $[\mathrm{CTA}] /[\mathrm{I}]=10 / 1$ system to investigate the LVE within the sol-gel transition (Scheme 1 and Table 1). Acrylic thymine with flexible spacer was chosen so that the stickers have sufficient freedom to associate/dissociate. ${ }^{10}$

Table 1. Molecular Characterization of the (Co)polymers

\begin{tabular}{lcccc}
\multicolumn{1}{c}{ sample } & $\begin{array}{c}\text { sticker }^{a} \\
(\mathrm{~mol} \%)\end{array}$ & $\begin{array}{c}\text { no. of stickers per } \\
\text { chain }^{a}\end{array}$ & $\begin{array}{c}\bar{M}_{\mathrm{n}}{ }^{a} \\
(\mathrm{~kg} / \mathrm{mol})\end{array}$ & $\begin{array}{c}\bar{M}_{\mathrm{n}, \mathrm{stick}}{ }^{b} \\
(\mathrm{~kg} / \mathrm{mol})\end{array}$ \\
PnBA & 0 & 0 & 24 & \\
PTHY10 & 8 & 18 & 32 & 1.8 \\
PTHY30 & 32 & 43 & 26 & 0.6 \\
PTHY100 & 100 & 159 & 51 & 0
\end{tabular}

${ }^{a}$ Estimated from ${ }^{1} \mathrm{H}$ NMR. ${ }^{b}$ Average molecular weight between the stickers in the copolymers.

${ }^{1} \mathrm{H}$ NMR analysis of the products (Figure 1 and Figures $\mathrm{S} 1-$ S4) proves the formation of the (co)polymers. The peak at $11.20 \mathrm{ppm}$, which belongs to the $-\mathrm{NH}$ proton shows two peaks adjacent to each other. This can be due to hydrogen bonding between - NH hydrogen donor and two different hydrogen bond acceptors, leading to two different chemical shifts: one with DMSO (solvent) and another one with $\mathrm{C}=\mathrm{O}$ in thymine moiety. By comparing the relative ratio of these two peaks, a transition can be seen with a change in sticker composition such that the left peak diminishes and the right peak enlarges (Figure S5). It can be a signature of intramolecular association as the amount of thymine is significantly increased to one per repeating unit and consequently the probability of intramolecular association enhanced remarkably. Further analysis, unfortunately, failed due to very low solubility or insolubility of the polymers in other solvents or lower temperatures.

For the prepared (co)polymers with different thymine contents the linear viscoelasticity (LVE) was investigated via melt rheology, and the corresponding master curves were built

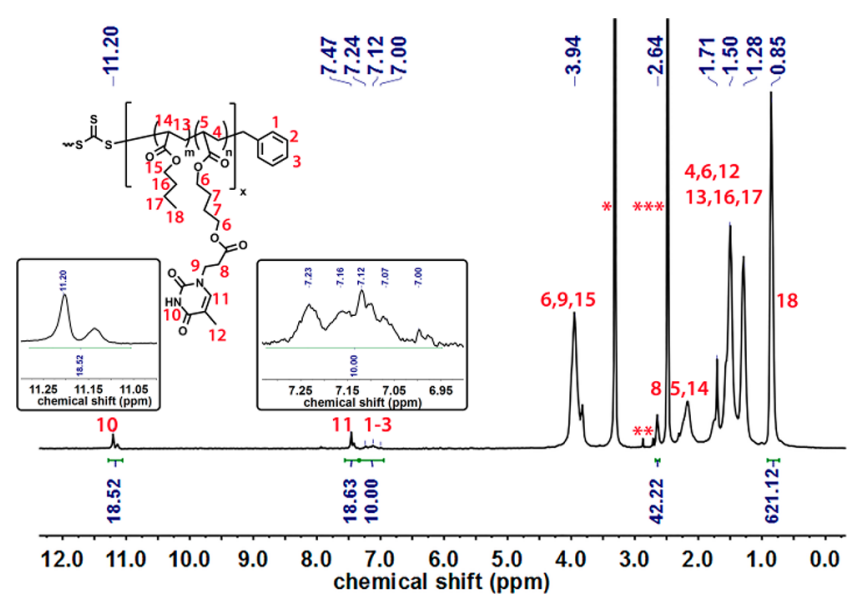

Figure 1. ${ }^{1} \mathrm{H}$ NMR spectra of PTHY10 in DMSO- $d_{6}$. *water residue, $* * \mathrm{DMF}$ residue, and $* * * \mathrm{DMSO}-d_{6}$.

experimentally by horizontally shifting of the frequency sweeps by a shift factor $a_{T}$, following a Williams-Landel-Ferry (WLF) equation, referenced to $T_{r}=T_{g}+40{ }^{\circ} \mathrm{C}$ (Figure 2). Thermorheologically complex (TRC) behavior was observed for samples containing stickers, in particular for PTHY100 (see Table 1). This is a well-known behavior for sticker-containing polymers $3,8,15,16,20,21$ originating from different temperature dependence of $\tau_{s}$ and $\tau_{0}$ (relaxation time of one Kuhn segment):

$$
\tau_{s}=\tau_{0} \exp \left(E_{a} / k T\right)
$$

where $E_{a}$ stands for the activation energy of the sticker dissociation. ${ }^{3}$ At high temperature $\left(75,85\right.$, and $\left.90{ }^{\circ} \mathrm{C}\right)$, the terminal relaxation of PTHY100 becomes visible and the relaxation time (controlled by $\tau_{s}$ ) shifts to higher frequencies with temperature increase, indicating an accelerated sticker dissociation in comparison to the Rouse-type motion (Figure 2d).

On the other hand, considering only the high frequency part of the storage modulus, time-temperature superposition ( $t T s)$ is fairly satisfied. This can be explained by using the Maxwell model $G^{\prime}(\omega)=\sum_{p} \omega^{2} \tau_{p}^{2} /\left(1+\omega^{2} \tau_{p}^{2}\right)$ and $G^{\prime \prime}(\omega)=\sum_{p} \omega \tau_{p} /(1$ $+\omega_{2} \tau_{p}^{2}$ ), in which $G^{\prime}$ scales with $\tau_{p}^{2}$ (sensitive to slow modes) and $G^{\prime \prime}$ scales with $\tau_{p}$ (sensitive to fast modes). ${ }^{8}$ In consequence, $G^{\prime}$ is more sensitive to the sticker dissociation (slow modes of relaxation) at low frequencies, and a more noticeable TRC is observed. While in Figure 2 the constructed master curves are based on the shift factors $a_{T}$, we now build these last ones only based on $G^{\prime}$ data using different shift 
(a)

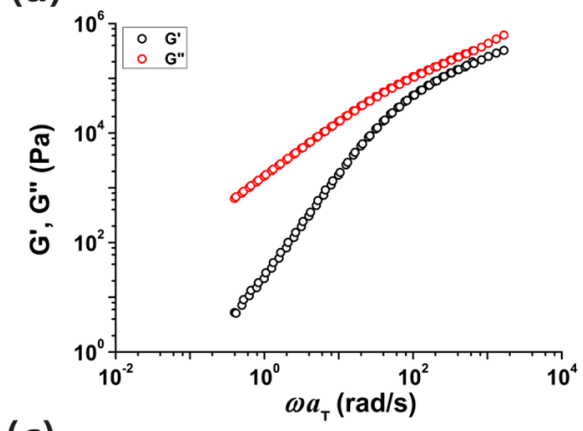

(c)

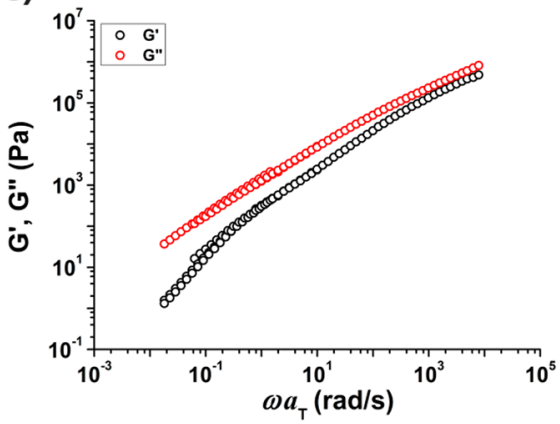

(b)

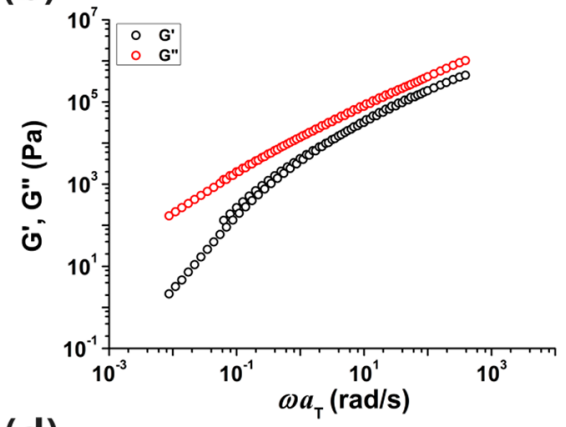

(d)

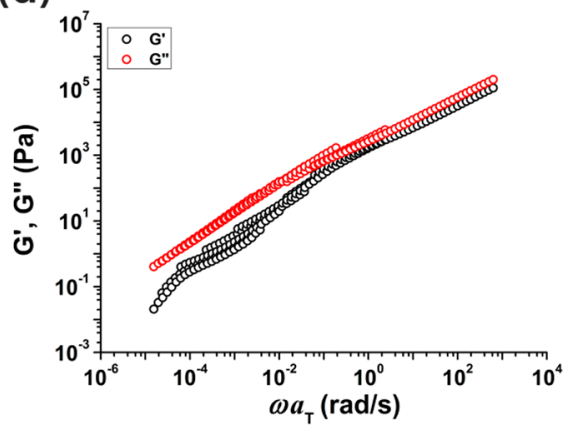

Figure 2. Test of $t$ S s of the storage and loss moduli for (a) PnBA, (b) PTHY10, (c) PTHY30, and (d) PTHY100, referenced to $T_{r}=T_{g}+40{ }^{\circ} \mathrm{C}$.

factors $a_{T}^{\prime}$, such that $t T s$ can be satisfied. Figure 3a shows the master curves ending up with a terminal slope close to 2 . At highest frequencies Rouse-like behavior is observed for all samples (zone I; black line). In the case of the reference

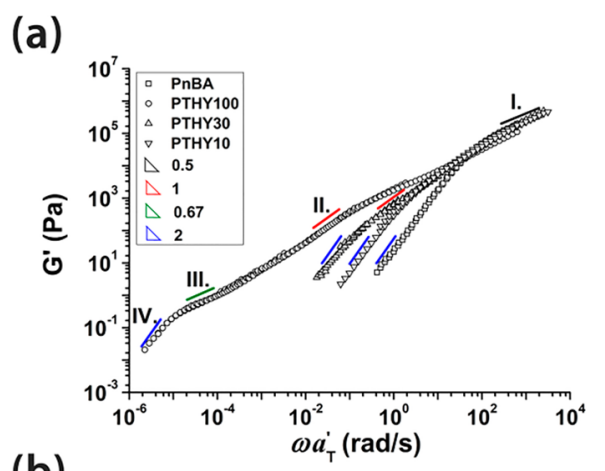

(b)

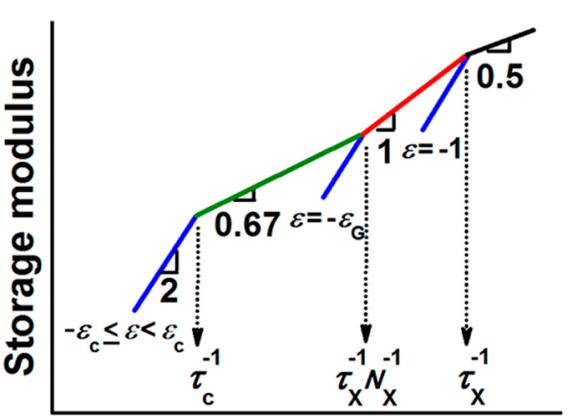

Frequency

Figure 3. (a) Master curves constructed for PnBA, PTHY10, PTHY30, and PTHY100 referenced to $T_{r}=T_{g}+40{ }^{\circ} \mathrm{C}$. (b) Summary of the modified MF percolation theory, including the Ginzburg transition and critical percolation regime. ${ }^{3}$ sample without sticker the terminal relaxation is subsequently visible with a slope close to 2 , corresponding to the Maxwell relaxation (zone IV; blue line). This indicates that PnBA as expected is within the first regime $(\varepsilon=-1)$. For PTHY10, zone II (red line) is observed in the intermediate frequency $(\omega$ $\sim 1-100 \mathrm{rad} / \mathrm{s})$, which is further extended to lower frequencies $(\omega \sim 0.1 \mathrm{rad} / \mathrm{s})$ for PTHY30 with a slope close to 1 . This is indicative of a cluster formation expressed by the MF percolation $\left(-1<\varepsilon<-\varepsilon_{\mathrm{G}}\right.$ ) (Figure $3 \mathrm{~b}$ ). With increased sticker concentration to $100 \%$, a fourth region is observed (green line) showing a slope of around 0.67 , covering 1 decade of the master curve just before the terminal relaxation and showing a transition from MF percolation to the critical percolation (Ginzburg point) and $-\varepsilon_{\mathrm{G}} \leq \varepsilon<\varepsilon_{\mathrm{c}}$. It has to be noted that the assignment of this regime to $-\varepsilon_{\mathrm{G}} \leq \varepsilon<\varepsilon_{\mathrm{c}}$ is solely based on the slope of 0.67 and the location just before the terminal relaxation, and it does not necessarily show that $-\varepsilon_{\mathrm{c}} \leq \varepsilon$ (in the effective breakup region) as these two regimes $\left(-\varepsilon_{\mathrm{G}} \leq \varepsilon<-\varepsilon_{\mathrm{c}}\right.$ and $\left.-\varepsilon_{\mathrm{c}} \leq \varepsilon<\varepsilon_{\mathrm{c}}\right)$ show a common theoretical slope 0.67 and hard to distinguish between them only based on the slope values. The storage modulus in this region has contributions from the segmental motion of the precursor chain in zone I (black line in Figure 3a,b), Rouse relaxation of the cluster formed within MF percolation regime in zone II (red line), Rouse relaxation of the entire cluster formed by critical percolation in zone III (green line), and finally the Maxwell type terminal relaxation of the cluster (blue line). ${ }^{3}$ The larger size of the zone II compared to the zone III refers to the domination of MF percolation, which is consistent with the fact that chains are overlapping (MF assumption) with $N=$ 159. The lifetime of the cluster is much longer than $\tau_{X}$ observable from the $\tau_{\text {char }}$ which goes to very low frequencies. The modulus values corresponding to zone II (and III) are much lower than $v k T\left(=\rho R T / M=5.5 \times 10^{4} \mathrm{~Pa}\right)$, which verifies that the system is well below the full gel point. These values are surprising as $p_{c}=0.006$ and $\varepsilon=166 \gg 1$ for 

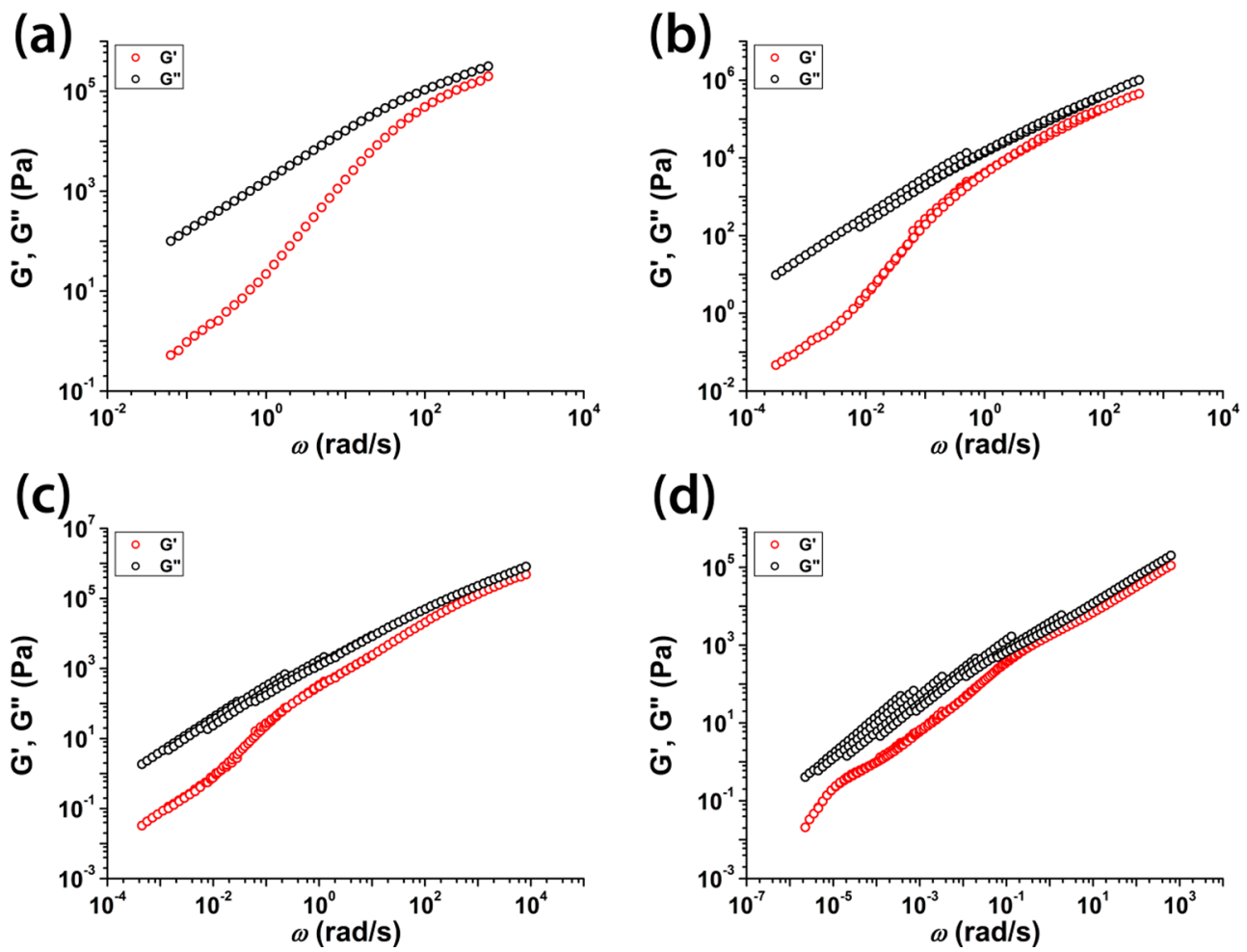

Figure 4. Full master curves for (a) PnBA, (b) PTHY10, (c) PTHY30, and (d) PTHY100, referenced to $T_{r}=T_{g}+40{ }^{\circ} \mathrm{C}$.

PTHY100. On the other hand, no plateau was observed, which means only few stable stickers with long lifetime are present, and the chains relax as an ensemble of linear polymers (sol) and clusters (gel). The fact that only a fraction of the weak stickers are associated (no network can be formed even with the expense of full functionalization of the precursor polymer, PTHY100), but they stay active leading to a cluster stable even until very low frequencies (long times), has important implications; being a weak hydrogen bonding does not necessarily mean a fast destruction of the network, but it means that the amount of active moieties is much less than a covalent analogue. Therefore, strength in this sense is a thermodynamic character, with weak hydrogen bonding having low equilibrium constant $K_{a} \sim \frac{k_{\text {ass }}}{k_{\text {diss }}}\left(\sim 70 \mathrm{M}^{-1}\right)^{22}$ and both $k_{\text {ass }}$ and $k_{\text {diss }}$ are either both low or both are equally high so as to keep $K_{a}$ (their ratio) low. As $k_{\text {diss }} \sim 1 / \tau_{s}$ long lifetime of the cluster can suggest a small $k_{\text {diss }}$ and $k_{\text {ass. }}$. This implies little tendency for association (no network formation) as well as dissociation (long-lasting cluster).

In order to have more insight into the kinetics, we use the two sets of shift factors $a_{T}$ and $a_{T}^{\prime}$ to obtain the activation energy of dissociation $E_{a}$ (Figure S6). At low temperatures, both shift factors overlap, which shows the contribution from the Rouse-type motion. However, by increasing temperature, they diverge and $a_{T}^{\prime}$ no longer follows the WLF behavior; instead, it shows Arrhenius dependence with a corresponding activation energy of $E_{a}=163 \mathrm{~kJ} / \mathrm{mol}$. However, this value does not reflect the dynamics of the stickers, as it was recently shown by Chen et al., ${ }^{3,8,14}$ who studied the associative energy in strongly associating polymers, such as PnBA functionalized with 2-ureido-4(3H)-pyrimidinone (Upy) and sulfonated polystyrene (SP). The authors showed that based on eq 7, a much lower value of $E_{a}$ was found (33 and $70 \mathrm{~kJ} / \mathrm{mol}$ for PnBA-UPy and SP8 compared to $75-120$ and $177 \mathrm{~kJ} / \mathrm{mol}$ with the $a_{T}^{\prime}$ method). ${ }^{5-7,9}$ This inconsistency was explained by the fact that $E_{a}$ based on eq 7 corresponds to $\tau_{s} / \tau_{0}$, whereas using $\ln \left(a_{T}^{\prime}\right)$ vs $1 / T$ the effect of segmental dynamics (related to $\left.\tau_{0}\right)$ is not excluded. Therefore, an accurate method to calculate $E_{a}$ is to use $a_{T}^{\prime} / a_{T}$, which is proportional to $\tau_{s} / \tau_{0}$. Using this strategy, we obtain $52 \mathrm{~kJ} / \mathrm{mol}$, which is much closer to 33 and $70 \mathrm{~kJ} / \mathrm{mol}$ obtained for PnBA-UPy and SP from $\tau_{s} /$ $\tau_{0}$ (eq 7) and $37 \mathrm{~kJ} / \mathrm{mol}$ for PnBA-UPy based on $a_{T}^{\prime} / a_{T}{ }^{8}$. There is still one question left: with such a high $E_{\mathrm{a}}(52 \mathrm{~kJ} / \mathrm{mol}$ $\gg k T$ ) large fractions of the stickers should be associated, so why can no plateau be seen in the corresponding master curve (Figure 3a)? In order to explain this apparent contradiction, it should be noted that despite the weakness of the stickers they are abundant. Anthamatten et al. $^{9}$ investigated the $E_{a}$ of polymers based on $\mathrm{AA}, \mathrm{AP}$, and $\mathrm{CEA}$ and realized that increasing the sticker concentration leads to an increase in $E_{a}$, while no plateau referring to network formation was observed. These findings indicate that high concentrations of weak hydrogen bondings are not equivalent to small number of strong stickers. This revelation is applicable to PTHY100 with high $E_{a}$ in which the stickers are distributed along the entire polymer; i.e., weak hydrogen bondings have a much wider distribution of energy, which may lead to a lack of network formation. However, another explanation would be the presence of multiple hydrogen bonding for PTHY100, which may further broaden the power-law region. Another plausible explanation considering the ${ }^{1} \mathrm{H}$ NMR analysis (Figure S5) can be the presence of intramolecular associations. With increasing the sticker concentration, although $\varepsilon$ increases the probability of intramolecular associations also increases as was confirmed with ${ }^{1} \mathrm{H}$ NMR investigations, preventing the chains to form a network.

An important difference between weak and strong stickers in general is that with increasing temperature the fraction of weak stickers that are in the associated state can change whereas it is 
not the case for strong hydrogen bonding moieties. This can influence the values obtained for the activation energy. Nevertheless, Figure $2 \mathrm{~d}$ shows that this effect is not significant, besides considering that the majority of the stickers must be associated leading to a cluster relaxation as was observed in Figure 3a.

It has to be noted that after the terminal slope of close to 2 for samples PnBA, PTHY10, and PTHY30, they do not fully relax and a shoulder is observed if the data regarding lower frequencies is considered, as indicated by a $G^{\prime}$ slope slightly lower than 2 (Figure 4). The absence of terminal slopes of 2 and 1 for $G^{\prime}$ and $G^{\prime \prime}$ in random copolymers bearing stickers has been ascribed to different phenomena: (1) a random distribution of the stickers along the chain, (2) a disproportionate amount of stickers in different chains, and (3) polydispersity in the chain length, leading to a distribution of terminal relaxation times. ${ }^{18,19}$ However, this effect has been seen for unfunctionalized PnBA as well. ${ }^{16}$ For the case of PnBA it has been argued that the appearance of a slope smaller than 1 and 2 is due to the presence of acrylic acid groups formed via accidental hydrolysis during the synthesis of PnBA. On the other hand, the presence of nanodomains and nanophase separation in poly( $n$-alkyl acrylate)s (for alkyl groups larger than propyl, i.e., butyl, pentyl, etc.) has been reported earlier. ${ }^{17}$ Because of the incompatible main chain and alkyl side chain, the alkyl nanodomains form aggregates with a typical size of 0.5-2 nm. Although the authors did not perform melt rheology to visualize this phenomenon, this explanation for the terminal shoulder in PnBA seems to be more plausible than partial hydrolysis of $n$-butyl acrylate groups to acrylic acid moieties. For the case of PTHY10 and PTHY30, both the sticker distribution and the presence of phase-separated acrylate moieties can be the reason for the smaller slopes than expected at the lowest frequencies. This is especially interesting when the master curve of PTHY100 is studied (Figure 4). A well-defined terminal relaxation was observed with $G^{\prime}$ values close to $0.01 \mathrm{~Pa}$. This implies that the arrangement of butyl groups is prevented by bulky thymine groups for PTHY100, and no phase separation can be seen in the corresponding master curve when the butyl acrylate composition is zero. Moreover, the sticker distribution is prevented when using fully functionalized polymer PTHY100. It has to be pointed out that even in the case of PTHY100 the terminal $G^{\prime}$ slope is not exactly 2 , which might be due to relative chain length distribution $(\boxplus)$ of 1.3 . Further analysis of phase separation requires X-ray analysis and will be the goal in our future studies; therefore, at this point only a primary conclusion can be made based on the melt rheology data.

\section{CONCLUSION}

By using different compositions of a flexible weak sticker along the polymer chain, LVE analysis showed a behavior remarkably close to the recently developed gelation theory for transient networks. The scaling revealed a transition from linear chains to a cluster, but any attempt to obtain a gel/network failed. First, by introducing one sticker per repeating unit of the precursor polymer, the effect of sticker distribution along the chain as well as phase separation is excluded, and then considering pure contribution from association of the stickers two conclusions were made: First, as the formed cluster was stable until very low frequencies (long time scales), this shows that weakly associated networks do not dissociate rapidly and can relax as a cluster at extended time before the dissociation of stickers can lead to the relaxation of the linear precursors (slow kinetics). Second, since the formation of gel was not possible even with the expense of a fully functionalized polymer chain bearing 159 stickers, this shows that the weakness of the stickers is their inability for complete association (thermodynamically not favored).

\section{ASSOCIATED CONTENT}

\section{Supporting Information}

The Supporting Information is available free of charge on the ACS Publications website at DOI: 10.1021/acs.macromol.8b00772.

Experimental procedures, ${ }^{1} \mathrm{H}$ NMR spectra, and the shift factors used for the master curves (PDF)

\section{AUTHOR INFORMATION}

\section{Corresponding Author}

*Tel +31-50 363 6867; e-mail k.u.loos@rug.nl (K.L.). ORCID ${ }^{\circ}$

Csaba Fodor: 0000-0002-5157-0535

Evelyne van Ruymbeke: 0000-0001-7633-0194

Katja Loos: 0000-0002-4613-1159

\section{Funding}

The research was supported by a NWO-VICI innovational research grant.

\section{Notes}

The authors declare no competing financial interest.

\section{ACKNOWLEDGMENTS}

The authors are grateful to Prof. Quan Chen (CAS), Dr. Salvatore Costanzo (UM), and Prof. Giuseppe Portale (RUG) for fruitful discussions. Evelyne van Ruymbeke is research associate of FNRS.

\section{REFERENCES}

(1) Herbst, F.; Dohler, D.; Michael, P.; Binder, W. H. Self-healing polymers via supramolecular forces. Macromol. Rapid Commun. 2013, 34 (3), 203-20.

(2) Li, J.; Lewis, C. L.; Chen, D. L.; Anthamatten, M. Dynamic Mechanical Behavior of Photo-Cross-linked Shape-Memory Elastomers. Macromolecules 2011, 44 (13), 5336-5343.

(3) Chen, Q.; Huang, C.; Weiss, R. A.; Colby, R. H. Viscoelasticity of Reversible Gelation for Ionomers. Macromolecules 2015, 48 (4), $1221-1230$.

(4) Goldansaz, H.; Voleppe, Q.; Pioge, S.; Fustin, C. A.; Gohy, J. F.; Brassinne, J.; Auhl, D.; van Ruymbeke, E. Controlling the melt rheology of linear entangled metallo-supramolecular polymers. Soft Matter 2015, 11 (4), 762-774.

(5) Feldman, K. E.; Kade, M. J.; Meijer, E. W.; Hawker, C. J.; Kramer, E. J. Model Transient Networks from Strongly HydrogenBonded Polymers. Macromolecules 2009, 42 (22), 9072-9081.

(6) Feldman, K. E.; Kade, M. J.; Meijer, E. W.; Hawker, C. J.; Kramer, E. J. Model Transient Networks from Strongly HydrogenBonded Polymers. Macromolecules 2010, 43 (7), 3576-3576.

(7) Feldman, K. E.; Kade, M. J.; Meijer, E. W.; Hawker, C. J.; Kramer, E. J. Model Transient Networks from Strongly HydrogenBonded Polymers. Macromolecules 2011, 44 (13), 5537-5537.

(8) Zhang, Z.; Liu, C.; Cao, X.; Gao, L.; Chen, Q. Linear Viscoelastic and Dielectric Properties of Strongly Hydrogen-Bonded Polymers near the Sol-Gel Transition. Macromolecules 2016, 49 (23), 9192-9202.

(9) Lewis, C. L.; Stewart, K.; Anthamatten, M. The Influence of Hydrogen Bonding Side-Groups on Viscoelastic Behavior of Linear and Network Polymers. Macromolecules 2014, 47 (2), 729-740. 
(10) Cheng, S.; Zhang, M.; Dixit, N.; Moore, R. B.; Long, T. E. Nucleobase Self-Assembly in Supramolecular Adhesives. Macromolecules 2012, 45 (2), 805-812.

(11) Rubinstein, M.; Semenov, A. N. Thermoreversible Gelation in Solutions of Associating Polymers. 2. Linear Dynamics. Macromolecules 1998, 31 (4), 1386-1397.

(12) Rubinstein, M.; Semenov, A. N. Dynamics of Entangled Solutions of Associating Polymers. Macromolecules 2001, 34 (4), 1058-1068.

(13) Chen, Q.; Tudryn, G. J.; Colby, R. H. Ionomer dynamics and the sticky Rouse model. J. Rheol. 2013, 57 (5), 1441-1462.

(14) Zhang, Z.; Huang, C.; Weiss, R. A.; Chen, Q. Association energy in strongly associative polymers. J. Rheol. 2017, 61 (6), 11991207.

(15) Hawke, L. G. D.; Ahmadi, M.; Goldansaz, H.; van Ruymbeke, E. Viscoelastic properties of linear associating poly(n-butyl acrylate) chains. J. Rheol. 2016, 60 (2), 297-310.

(16) Shabbir, A.; Goldansaz, H.; Hassager, O.; van Ruymbeke, E.; Alvarez, N. J. Effect of Hydrogen Bonding on Linear and Nonlinear Rheology of Entangled Polymer Melts. Macromolecules 2015, 48 (16), 5988-5996.

(17) Beiner, M.; Huth, H. Nanophase separation and hindered glass transition in side-chain polymers. Nat. Mater. 2003, 2, 595.

(18) Seiffert, S. Effect of Supramolecular Interchain Sticking on the Low-Frequency Relaxation of Transient Polymer Networks. Macromol. Rapid Commun. 2016, 37 (3), 257-264.

(19) Shabbir, A.; Javakhishvili, I.; Cerveny, S.; Hvilsted, S.; Skov, A. L.; Hassager, O.; Alvarez, N. J. , Linear Viscoelastic and Dielectric Relaxation Response of Unentangled UPy-Based Supramolecular Networks. Macromolecules 2016, 49 (10), 3899-3910.

(20) Ahmadi, M.; Hawke, L. G. D.; Goldansaz, H.; van Ruymbeke, E. Dynamics of Entangled Linear Supramolecular Chains with Sticky Side Groups: Influence of Hindered Fluctuations. Macromolecules 2015, 48 (19), 7300-7310.

(21) Chen, Q.; Colby, R. H. Linear viscoelasticity of sulfonated styrene oligomers near the sol-gel transition. Korea-Australia Rheology Journal 2014, 26 (3), 257-261.

(22) Herbst, F.; Binder, W. H. Comparing solution and melt-state association of hydrogen bonds in supramolecular polymers. Polym. Chem. 2013, 4 (12), 3602-3609. 